\title{
Universal Properties of Human Language in the Light of Natural Phenomena
}

\author{
Farman Zeynalov ${ }^{1}$ \\ ${ }^{1}$ Azerbaijan University of Languages, Baku, Azerbaijan \\ Correspondence: Farman Husan Zeynalov, Department of English Phonetics, Azerbaijan University of Languages, \\ Baku, Azerbaijan. E-mail: Zeynalov.farman@adu.edu.az
}

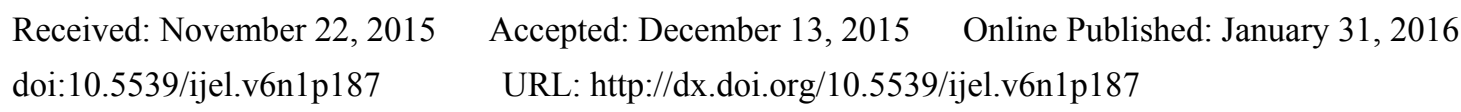

\begin{abstract}
The investigations of linguistic literature show that linguists today are much inspired by the goals of generative grammar, as well as the important human universals which explain child language acquisition, the universals of general cognition and learning. To put it the other way, linguists are interested in studying the human language to discover the nature of Universal Grammar the principles of which characterize all human languages and this major aim becomes the basis of contemporary linguistic theory of today. As D. Crystal writes: " $\ldots$ the main task of the linguistic scholar is basically to study and understand the general principles upon which all languages are built" (Crystal, 1977).

In this respect we are sure that this article may throw some light on the origin of universal properties of human language.

Our aim, accordingly, is to study the origin of general principles of human language and to discover natural factors which cause the human language to have universal properties. In other words, our aim is "to find out the laws of human language" in relation to natural phenomena. In this connection an attempt is made to describe variety of observable similar facts and compare them both pertaining to human language and natural processes.
\end{abstract}

Keywords: isomorphism, unconsciousness, code, common features, system, structure, rhythmic structure, correlates, nucleic acids, complimentary principle, hierarchy, impinge, inherit, transform, geological processes, phylogenesis

\section{Introduction}

The theoretical background of the article is based on some considerations which were underlined in the works of distinguished linguists (R. Jacobson). Thus, it was revealed that genetic code resembles linguistic code from structural and functional point of view. It should be noted that the fact of unconsciousness is not only the fact that is expressed in structural mechanism of language on the model of the genetic code but it is also observed in different creative activities, such as in ancient Chinese philosophers special system of transformation of four binary elements, N. Y. Marr's theory of glottogonic processes, Hippocrate's system with four liguids (Gamkralidze, 1988).

The act of unconsciousness further is observed in creative activity of ancient craftsmen who created geometrical ornaments imitating the structural formation of crystal substances. The act of consciousness is also observed in weaving of carpets, rugs etc. (Mammadov, 1981). The act of consciousness can be found in "creative activity" of bees and spider whose honeycomb and cobweb resemble rhythmically arranged geometrical figures. The similarity between language and geology were underlined and became unexpected to the participants of the conference held in Bad Homburg (Germany) in 1989 which dedicated to the problem of "Language and earth" (Jacob, 1977).

Thus, without going into detail we may say that there is close connection between the general law of human language and geological, biological processes.

\section{Research Methodology}

We have used in this article direct observation, structural isomorphism, comparative, linguistic description, transformation, logical-heuristic methods. 


\section{Scope of the Study}

The problem of isomorphism of genetic and linguistic codes has remained in the focus of attention of Roman Jacobson for a long time. (6) He proposed an original solution of this problem which emerged in the juncture of comparatively new scientific disciplines - semiology and molecular genetics. The discovery of the mechanism of heredity in molecular biology revealed that heredity corresponds to the information written along chromosomes with a definite kind of chemical "alphabet".

The initial elements or "letters" of this alphabet consist of four chemical radicals (adonin, timin, sitozin, guanin) combined with one another in an endless linear sequence of nucleic acids and create a kind of chemical text of genetic information. Similarly, the sentence is the segment of a definite linguistic text composed of linear sequences of a small number of discrete units - phonemes or letters. In genetic code a separate element of the system is not informative, certain information is composed of special combinations of four initial elements (nucleotides), three of which create the so-called "triplets". Similar to this, in linguistic code the initial units-phonemes are meaningless in isolation, they serve as the basis for organization of definite minimal consequent linear combinations which express a certain content within a given system.

In genetic code the four initial elements in threes make up totally 64 combinations, i.e. the genetic code consists of 64 words out of which three triplets present themselves as "punctuation marks", which identify the beginning and the end of the "sentence" in the long sequence of nucleic acids, and the rest correspond to one out of 20 aminoacids.

Thus, it was revealed that genetic code resembles linguistic code from structural and functional point of view, and it is not accidental that at the very beginning of deciphering genetic code, molecular genetics began to borrow linguistic notions and terminology. At the same time there are cases in linguistics when it also borrows notions and terms from molecular genetics.

The distinguishing feature between linguistic code and genetic code is the number of initial units-phonemes. The combination of phonemes constitutes minimal significant units in language, and this creates redundancy in language which allows correcting or restoring any distortion in the information arising as a result of infringement under the influence of external factors. But the genetic code does not possess such characteristics, any mutation or elimination of separate elements in the linear sequence of nucleotides leads inevitably to the distortion of the originally written genetic information.

The structural isomorphism revealed between two different information systems, i.e. genetic and linguistic, built on linear combination of source discrete units, gives rise to a question concerning the nature of these systems and the reasons of their similar structural isomorphism. In this connection different views have been put forward, and among them the argument between R. Jacobson, a linguist, and F. Jacob a biologist genetic, is very characteristic in this respect. The essence of their argument is whether the structural isomorphism revealed between genetic and linguistic codes is purely external arising as a result of structural similarity of two different systems which perform informative functions, or this isomorphism is a result of phylogenetic formation of linguistic code. R. Jacobson maintains the second point of view, while F. Jacob the first one. Jacobsonian understanding of the structural isomorphism of genetic and linguistic codes presupposes evolutionary process of superposition of linguistic code directly on genetic one, imitating its structural principles which are realized on condition of unconsciousness. The fact of unconsciousness is not only the fact that is expressed in phylogenetic process of mounting of the structural mechanism of language on the model of genetic code but it is also observed in different creative activities. In this connection we can remember some incidents concerning the act of unconsciousness. N. Y. Marr's theory of glottogonic process is based on intuitive considerations whose peculiar scientific intuition allowed him to come to quite unexpected conclusion. According to Marr the vocabulary stock of diverse languages arising historically is based on four initial elements consisting of peculiar sound triplets each (sal, ber, yon, rosh) which is meaningless in triplicate sequence, and any text of arbitrary length in any language of the world is a result of phonetic reorganization of these four elements.

Ancient Chinese philosophers about three thousand years ago compiled a book bearing the title "Chinese Book I Ching. The Book of Transformation" and worked out a special system of transformation of four binary elements made out of "the male principle" Yang and "the female principle" Yin and grouped in threes which gives totally 64 triplicate sequences what is analogical to genetic code. By means of this symbolic system all diverse living things were described and brought into correlations. In this context especially significant are considerations of the system with four elements of the universe in cosmogony of Ionia school, and Hippocrates' system with four liquids (yellow bile, black bile, blood and phlegm) of the human body. 
The act of unconsciousness is also observed in creative activity of ancient art craftsmen who created geometrical ornaments imitating the structural formation of crystal substances. This fact became known to science after studying the structure of crystal by the X-ray structural analysis method. At the International Congress of Crystallographers in Warsaw (1978) the well-known Azerbaijani scientist Khudu Mammadov proved that the art style which Morris Escher revived in 1920 and which had been widely used in the works of ancient craftsmen is based on the laws and principles of crystallography. It was revealed that Escher and his predecessors in their works unconsciously used mathematical model of crystal i.e. they arranged their ornaments on the plane in the same way as nature arranges molecules in crystal substances (Mammadov, 1981).

Similar facts can be found in "creative activity" of bees. Bees build their cells in a honeycomb in accordance with the structure of aroma or odor, i.e. directly imitating the chemical structure of aromatic carbonhydrogens whose structure is hexagonal geometrical figure which is identical with the shape of the bee cells, i.e. both are in the form of hexagonal geometrical figures. The structural formation and principle of arrangement of bee cells are much similar to crystal ornaments whose cells recur periodically in a honeycomb with the same shape. A spider spins its cobweb in the shape of geometrical figure. The cobweb of the spider consists of the same rhythmically arranged geometrical figures and though they are different in dimensions they are also similar to crystal ornaments.

The connection of language with a living organism is a generally known fact, but the facts revealing similarity between language and geology were unexpected to the participants of the conference held in Bad Homburg (Germany) in 1989 and dedicated to the problems of "Language and earth: elective affinities between the emerging sciences of linguistics and geology". Relying on a number of revealed facts, the scientists proved that there are many common features between linguistics and geology. They refer these facts to the interest of scholars. Thus the founders of modern geology A. G. Werner and J. Hatton were also interested in linguistics, at the same time Whitney brothers, J. D. Whitney and W. D. Whitney, maintained close contact with their colleagues geologists. The scholars analyzed and discussed different points of view of the specialists of the $18^{\text {th }}$ and $19^{\text {th }}$ centuries on the structural similarity and integrity between language and geology, chemistry, biology. They accentuated an idea that scientific achievements in the field of physical sciences had played an important role for anthropology and linguistics. In the $19^{\text {th }}$ century the realistic picture of chronological periods of development of the earth was formed and on the basis of this fact of geology some linguists (Y. Adelung \& J. Grimm) considered it to be their main task to explain the development of language via the development of all mankind but with some geographical limitations. The others tried to explain the history of the earth and the origin of language, and the reasons that cause geographical and language changes on the basis of the catastrophy theory and the conception of uniformism. Furthermore, some scholars approached language and the Earth as a cyclic-working mechanism which evolves in the shape of a spiral realizing on the basis of antagonistic processes, destructive and creative forces.

In his article "W. D. Whitney and the influence of geology on the theory of linguistics" K. Kerner analyses and compares the works of the American linguist W. D. Whitney with the work of the English geologist Ch. Lyell and lays a special stress on the fact that W. D. Whitney did as much for linguistics as Ch. Lyell did for geology and that Whitney used Ch. Lyell's ideas and approached them somewhat critically. He introduced the notion of "uniformism" into the scientific use and played an important role in the history of linguistic theory and influenced Junggramatiker and Ferdinand de Saussure (4). In addition to this, we may say that the forms of language and the notions of two forces, "inner" and "outer", which occupy one of the central places in W. D. Whitney's linguistic teaching, are based on geological considerations. The two notions "inner" and "outer", which were introduced by W. D. Whitney, to our mind, correspond to Chomskyan theory of "deep" (inner) and "surface" (outer) structures.

In the $19^{\text {th }}$ century chemical molecules were regarded as an example of non-mechanical unity in which they are interconnected and interdependent. In accordance with this F.de Saussure determined the value of linguistic elements in the structure of language and by analogy introduced the notion of "valency" into linguistics, while A. Schleicher introduced the notion of "morphology" based on biological consideration, and W. D. Whitney-the notion of "two forces" and "uniformism", which in our opinion, is based on geological considerations and principles.

F.de Saussure himself states that not only in linguistics, generally, but studying the object of all sciences in detail two axes of research direction must be distinguished. In this respect one can find similarity between language and the earth. Lingual diachrony means different time-periods in the development of the lingual elements as well as language as a whole, which corresponds to different time-periods of the development of the strata of the earth. What is meant by lingual synchrony is coexistence of lingual elements at a particular time-period, i.e. in their static form, which corresponds to relief shape of the surface of the earth at every stage of its history. 
The notions of "internal" and "external" linguistics correspond directly to endogenous and exogenous processes or forces, and the linguistic terms of "paradigmatics" and "syntagmatics", which express two fundamental types of relation between lingual elements, can also be taken as similar to the changes that take place in inner and outer parts of the earth. The changes occur vertically and horizontally. The vertical changes take place in the bottom or depth of the earth and occur very slowly. This parameter corresponds to paradigmatics, i.e. to linguistic changes as a whole and they are usually slow and gradual. The horizontal changes occur on the surface of the earth and cause substantial changes on its relief. This parameter corresponds to syntagmatics where changes are rapid.

Moreover, one can observe the similarity between "The Table of the Periodic System of Chemical Elements" and paradigmatic and syntagmatic relations of lingual elements. Thus the chemical elements, which possess the same properties, occupy the vertical line and correspond to paradigmatics, and the chemical elements, which possess different properties, occupy the horizontal line, and that corresponds to syntagmatics.

\section{Isomorphism in Crystals, Genetic and Linguistic Codes}

The stratification model of linguistic system which was introduced into linguistics by the American scholars S. Lemm and H. A. Gleason can also be taken as similar to geological systems. What is meant in both models is the sequence and placement of geological and linguistic systems and that corresponds to the hierarchy of linguistic units. But today, the structural, as well as functional similarity between language and geology, rather crystals, is more evident. As it was mentioned earlier, when studying the structure of crystal substances, the Azerbaijani scientist Khudu Mammadov revealed that there exist common features and principles between the structural formation of crystals and ornaments, designs, as well as the patterns of geometric designs which have been utilized for centuries in architecture, design, minor arts, as well as weaving of carpets, rugs and so on (Mammadov, 1981).

It was revealed that the structure of crystals is formed by its elements (atoms and molecules) which recur in the bottom of crystals periodically, and such periodic recurrence in crystals create multistratum, self-creation and imagination of endlessness. The structure of language (speech) is formed by its elements (units) which recur periodically in different positions and such recurrences, as in crystals, create multilayers or levels and self-creation and imagination of endlessness or endless structures due to which numerous words and word combinations may be formed, and sentences are constructed, by means of which any thought and different variants of thought may be expressed, or putting this another way, "Language makes infinite use of finite means".

The similarity between crystal and language can also be observed in the articulatory configurations of speech sounds, rather vowels, whose articulatory areas are described in geometrical figures, the one of which was introduced by the English phonetician D. Jones in the form of a trapezium, the other by the Russian scholar L. V. Shcherba in the form of a triangle, and the Turkic-Azerbaijani vowels in a cube-shaped figure by J. Denny. Rhythm is one of the inherent features of crystal and language, whose elements recur periodically in their structure, creating rhythmical arrangement of their elements (Zeynalov, 1999).

There can also be observed functional similarity between crystal and language. Crystal substance performs two functions. Crystal substances are of biological and social importance by means of which human beings erect houses to live in and other buildings for different purposes to preserve life. Secondly, the structure of crystal substances, which present themselves as ornaments, are used for aesthetic purposes and information. Language performs the same functions that crystals do. Language serves as a means of communication conveying information by means of which human beings regulate their living and social needs, and through poetic and fiction forms of language, human beings regulate their emotional and aesthetic worlds (Zeynalov, 1999).

Proceeding from the above-mentioned similar parameters we may conclude that the fundamental notions which are widely used in linguistics, such as lingual diachrony, lingual synchrony, internal and external linguistics, paradigmatics, syntagmatics, and hierarchic relations between lingual units, are introduced into linguistics on the basis of geological considerations and principles.

So we may observe the following common features in crystals and genetic and linguistic codes.

The initial units of these systems consist of more or less discrete elements, i.e., crystal-atoms and molecules; genetic code-nucleotides, and linguistic code-phonemes. "Phonemes are like chemical elements in that they combine only with certain other elements" (Farb, 1977).

The structure of the three systems is formed by the recurrence of their elements which are arranged rhythmically, and rhythm is one of the inherent features of their structure.

In genetic and linguistic codes a separate initial element does not possess any meaning, neither does a separate cage of crystal perform an ornament or "information". The certain information is formed by the combinatory sequences of their elements. 
The three systems demonstrate the forms of a "triplet", i.e. the structural formation of crystal presents three dimensions, the units of language stand to one another in three types of relations, as for genetic code, it performs its informative function in peculiar combinations of three nucleotides.

The three systems are carriers of information, i.e. the different structure of crystal substances carries information on the climatic and rhythmic conditions of nature at a definite time - period, as well as various areas of it. Secondly, the structural shape of each crystal used as ornaments carries aesthetic information. Genetic code carries information about hereditary peculiarities of living beings; language -conveys information on history, culture and art of the human civilization. These systems, as a physical substance, accumulate and convey information from century to century, and hence present themselves as memory.

In these systems the sequence and placement of elements in the structures are based on the complementary principle, i.e. the appearance of the next element in the structure is determined by the previous one. For instance, if the first cage is known in the structure of crystal, the next cages will also be known. If in the chain of nucleic acids the initial nucleotide is adonin (A), the opposite nucleotide will be timin (T), and if it is sitozin (S), the opposite will be guanin $(\mathrm{G})$. To put it another way, if in the chain of nucleic acids the following sequences of nucleotides A,G,S,T,A,S,S occur, then in this case opposite complementaries will be T,S,G,A,T,G,G. At the same time the presence of the third nucleotide in the triplet is also determined by the complementary principle, whose presence in the sequence of triplets depends on preceding elements, i.e. if the preceding two nucleotides of the triplet are in sequence of S-G the third one will be $\mathrm{S}$, and the combinatorial sequence of nucleotides will be in the structure of S-G-S, and so on.

The complementary principle is the main characteristic feature of language which is observed in all languages at different levels. For example, it is one of the main principles in phonology, that is to say, the allophones of the same phoneme, unlike the phonemes, never occur in the same position and none of them can be used or never occurs instead of the other allophones. In the English language the agreement of the predicate with the subject, the forms of "to be", "to have", "to do", the plural forms of nouns-"s, es", etc, are based on complementary principle. In the Azerbaijani language the use of the front or back, soft or hard vowels in the suffixes is determined by the root vowel, and so on. All these common features existing among the three systems lead us to conclude that there is a structural and functional isomorphism among these systems, whose connections, or rather relations, seem to be natural but not accidental. As nature is the system of hierarchies where one system gives birth to another, i.e. systems are derived from one another on the principle of "heredity". Though these systems are formed in different time-periods, they possess "hereditary" features, i.e. the laws and principles existing in initial systems, for instance, in crystals, they are traced in the next systems - biological and linguistic. As the linguistic system is formed after crystal and genetic systems, in language the structural, functional and rhythmical properties are traced hereditarily. So the structural model or pattern of language is a result of imitation of laws and principles of crystal and genetic systems in phylogenetic evolutionary processes which are realized on condition of unconsciousness.

It should be noted that the act of unconsciousness brings forth a series of questions: how does it happen that the structural model of crystals, as geometric figures, appear in ornaments, designs and minor arts, as well as weaving of carpets, rugs? How does it happen that there is rhythm within crystals; at first sight they seem to be dead and inanimate, the atomic molecular structure of which recurs periodically creating the imagination of endlessness. How does it happen that bees make their cells and spiders their cobwebs in the shape of rhythmically arranged geometrical figures. How does it happen that genetic code corresponds to crystals with its periodically recurrence of self-creation? How does it happen that complimentary principle existing in genetic code appears in linguistic code? How does it happen that the expression means of communication in society are mainly realized by vocal sounds? Furthermore, how does it happen that noun phrase (NP) in all languages occurs initially in the structure of the sentence and verb phrase (VP) follows it? How does it happen that all languages possess almost the same parts of speech and members of sentences? How does it happen that there are two kinds of words, notional and function or structural, which occur in the sentence structure of all languages? How does it happen that collocations like phonetics and phonology, system and structure, man and woman, father and mother, husband and wife, hen and chicken, sheep and lamb, mountain and valley, fire and water, and so on, have a stable word order in isolation as well as in sentences? How does it happen that the inventory of the phonemes in all languages is too limited in comparison with words, word combinations and sentences? How does it happen that the limited inventory of phonemes create or generate endless word combinations and sentences?

How does it happen that when a baby is born, the first act that he or she performs is crying with high pitch voice? How does it happen that all the children of the world, independent of their nationality, social class, geography or even intelligence, acquire language in a similar way? How does it happen that when children start uttering phrases 
they always use correct sentences, not incorrect ones, and produce their sentences on the basis of NP + VP structure? How does it happen that the first organ formed in the embryo of a baby is the ears?

It should be noted that the links of human beings with the universe are much deeper and more virtual than our considerations and in many cases these unobserverable and virtual links emerge in various ways or manners depending not on humans consciousness.

It should also be noted that to create diversity in order to make economy of atoms, elements and phonemes of a system and substitute this minority with the structure, is the main principle of nature. To put it another way, structure is one of the main principles or ways of creating diversities from the finite number of the elements of the systems. Thus minority of elements of a system leads to their universality on the one hand, and makes it easy to produce or build endless word combinations and sentences on the other hand. For instance, all the substances or matters that we can observe are built from atoms of more than hundred chemical elements. The atoms of chemical elements are built of three elementary high-energy particles: electron, proton and neutron. It is possible to construct or form all the words in all languages from about thirty five phonemes. All the colour variations in the eye are obtained from the mixture of three colours (Mammadov, 1981).

The nuclei acids which convey heredity peculiarities from generation to generation are formed by the four-based nitrous acid.

It seems unconceivable that seven notes are suffice to compose and write all the musical works.

It should be noted that there is similarity between the structural construction of atoms and the structure of a sentence. Molecules or complex atoms, consist of atoms, and the sentence consists of words, collocations, thoughts, and so on.

The noun phrase occurs in the sentence initially because substances have existed before the motion. Therefore, such kind of sequential arrangement occurs in the structure of any language.

Notional words name things and processes, their quality and quantity which refer to them. Function words in speech express relations between them. In this respect we may say that the word order in sentences and their relations between them is nothing else than the relation between objects and processes that are naturally sequenced and structured in the universe. The expressive means of language in society are the vocal sounds because the universe is itself noisy, and it has an analyzer in the human organism. It is the ears that perceive the acoustic information from the universe itself, as well as speech information in communication between the speaker and the hearer. In this respect $\mathrm{Ch}$. Hockett, to our mind, is quite right emphasizing the fact that sound change does go on because of noise. Only in a permanently noiseless universe there would be no events, hence no information, hence nothing to talk with. (7)

Being a part and parcel of the nature, that is to say, descending from the nature, the humans cannot create such a thing the prototype of which is not found in the nature or vice versa. For instance, the prototype of light existing in the universe is the eyes in a human being, the air which we breathe is the lungs, the lungs and the vocal cords, that produce sounds we hear, are the ears, the prototype of rhythm is the heart, and so on. Everything in the nature, both animate and inanimate, irradiate information which creates irritation, and this property of things and processes may be taken equal to the property of the human brain.

The human brain like things or processes in the nature irradiates information from itself in the form of thoughts.

In other words, the nature used its richest principles in the structure of the human body, and the humans can only utilize these principles. So the system and structure property of the nature are transformed into the human organism, whose whole system and structure consist of subsystems and substructures.

It should be pointed out that in order to consider investigation of an object as a system it should meet several requirements: the system should consist of lower or under systems; the system should constitute a part of a supreme system. Language as a system has lower, or under systems, such as the system of phonemes, morphemes, lexemes, phrasems, and so on.

If language is a system, then in this case it should constitute a part of a supreme system. To our mind, the supreme system of language is the universe. From this point of view W. Chafe's considerations on language are of great interest. To Chafe language is a set of habits relating with its vocal sounds with the universe... it impinges on non-linguistic reality in two ways; the vocal sounds, on the one hand, and the universe of things talked about, on the other hand... the study of all potential vocal properties is phonetics, while the study of all properties of the universe, that might be talked about, is semantics. Language selects properties from phonetics and semantics which are combined by language in various arrangements, the study of which constitutes phonology, when phonetic 
properties are involved; and grammar, when properties belong to semantics. Phonological and grammatical habits are independent of each other, but the two are linked by other complex habits the study of which is called morphophonemic (Chafe, 1962). To Louis Carini "But language just happened. It happened because language is the most natural outcome in a world of people where babies babble, and mothers babble back and where the baby also has the potential for metaphor." (Fromkin \& Rodman, 1988)

Thus, all principles, regularities and properties that exist in the nature are coded and put into nucleic acids by physical, chemical, biological, and psychological means and transformed or conveyed into the human organism unconsciously, and they are decoded when the human descends to society. Putting it another way, the human descends to society with a number of potential human ability, including language capacity, impinging upon the universe, that is to say, biological foundation, as well as universal properties of language are derived from the universe.

Proceeding from all above-mentioned considerations we may say that such aspects of language as phonology, grammar, semantics, as well as biological, psychological and creativity properties of language, in the common sense, have physical correlates in the universe.

So the vocal sounds, as physical correlate correspond to phonology; the structural arrangement of things and the relation between them - to grammar; the properties of things talked about - to semantics; the irradiation of information which creates irritation - to psychology; nucleic acids which convey hereditary information-to biology.

The physical correlate of creativity property of language corresponds to crystals, the elements of which create multistratum, self-creation and imagination of infinity.

\section{Conclusion}

Thus, the substantial study of the subject and analyzed issues allow us to make the following inferences:

Due to its many parameters, language behaves like natural objects, particularly like crystals which possess a certain rhythmic, regular structure, a kind of rhythmic property reiterated periodically. Thus, due to its systemic-structural nature, rhythm, structural differences, function, ability to bear, store and convey information, language much more resembles crystals and genetic code. This similarity shows that the isomorphism of language (speech) with geological processes, crystals and genetic code is not at the level of abstract notions; there are objective relations in their structures and common features in their origins. Though the birth and formation of these systems emerged in various periods of historical development, they conditioned the birth of each other; they inherited their peculiarities from each other. Therefore, the linguistic code, the structural model of language has emerged naturally in the process of phylogenesis evolution in compliance with the structural principles and structural models existing before them.

As the structural model of language has emerged after the birth of the two afore-mentioned systems, their structural principles and other properties are conspiucously observed in language. Thus, language remains a natural phenomenon in the chain of hierarchy of systems of the nature, a link in this chain with its own embryo, material basis and trace. Therefore, language should not be regarded as a pure social phenomenon. It should be regarded as innate-natural and social phenomenon, since its biological foundation and its universal properties derived from the universe.

\section{References}

Chafe, W. L. (1962). Phonetics, Semantics and Language. Language, 36(4), 335-344. http://dx.doi.org/10.2307/410669

Chomsky, N. (1968). Language and Mind. New York: Harcourt Brace.

Crystal, D. (1977). Linguistics. London: Penguin Books.

Farb, P. (1977). World Play. What happens when people talk. Caronet Books.

Fromkin, V., \& Rodman, R. (1988). An introduction to language (4th ed.).

Gamkralidze, Th. V. (1988). Jacobson and the problem of isomorphism between genetic code and semiotic systems. Issues of Linguistics, (3). Moscow.

Hockett, Ch. (1965). Sound Change. Language, 1(2), 185-204. http://dx.doi.org/10.2307/411873

Hudson, G. (2000). Essential introductory linguistics. USA: Blackwell Publishers. 
Jacob, F. (1977). The linguistic model in biology. In D. Armstrong \& C. H. van Schooneveld (Eds.), Roman Jacobson. Echoes his scholarship.

Jacobson, R. O. (1970). Linguistics and its relation to other science. In Main trends of research in social and human sciences. Part 1. Social sciences (pp. 419-463). Paris: The Hague, Moaton.

Jacobson, R. O. (1985). Selected works. Moscow.

Julla, S. F. (1978). Linguistics and language (2nd ed.). New York.

Kurilovich, Y. (1962). The notion of isomorphism. In Outlines of Linguistics. Moscow.

Mammadov, A.Y. (2008). Current issues in Linguistics. Baku.

Mammadov, X. et al. (1981). Memories of ornaments. Baku.

Naumann, B. P. H., Plank, F., \& Hofbauer, G. (1992). Language and earth: Elective affinities between the emerging sciences of linguistics and geology. Amsterdam, Philadelphia: Benyamins. http://dx.doi.org/10.1075/sihols.66

Zeynalov, F. H. (1999). Language and reality: system and structural relations. Baku.

\section{Copyrights}

Copyright for this article is retained by the author(s), with first publication rights granted to the journal.

This is an open-access article distributed under the terms and conditions of the Creative Commons Attribution license (http://creativecommons.org/licenses/by/3.0/). 